\title{
Expression of trophinin and dipeptidyl peptidase IV in endometrial co-culture in the presence of an embryo: A comparative immunocytochemical study
}

\author{
ELIF GELENLI DOLANBAY ${ }^{1}$, MELDA YARDIMOGLU ${ }^{1}$, ENDER YALCINKAYA ${ }^{2}$, \\ YUSUFHAN YAZIR $^{1,3}$, AYCA AKSOY $^{3}$, ERDAL KARAOZ $^{4}$ and ERAY CALISKAN ${ }^{5}$ \\ ${ }^{1}$ Department of Histology and Embryology; ${ }^{2}$ Training and Research Hospital, IVF Unit, Faculty of Medicine; \\ ${ }^{3}$ Center of Stem Cell and Gene Therapies Research and Practice, Kocaeli University, Campus of Umuttepe, Kocaeli 41380; \\ ${ }^{4}$ Center for Regenerative Medicine and Stem Cell Research and Manufacturing (LivMedCell), Liv Hospital, İstanbul 34340; \\ ${ }^{5}$ Department of Obstetrics and Gynecology, Faculty of Medicine, Bahçeşehir University, İstanbul 34353, Turkey
}

Received July 17, 2015; Accepted February 12, 2016

DOI: $10.3892 / \mathrm{mmr} .2016 .5020$

\begin{abstract}
Recurrent implantation failure leads to a reduced pregnancy rate. The expression patterns of trophinin and dipeptidyl peptidase IV (CD26) indicate the involvement of embryo implantation and early placental development. The purpose of the present study was to evaluate endometrial co-culture cells in the presence of embryo with trophinin and CD26 immunofluorescence staining. Patients with recurrent implantation failure were enrolled in the present study. The patients were aged between 26 and 36 years. Co-cultures were prepared from endometrial biopsies for each patient. Controlled ovarian hyperstimulation was performed on each of the patients. Certain embryos were maintained in a conventional culture environment $(n=80)$, and others in an endometrial co-culture environment $(n=25)$. Following embryo transfer, the co-culture cells were examined under an inverted wide-field fluorescence microscope. The ratio of a successful pregnancy was 0.38 in
\end{abstract}

Correspondence to: Professor Melda Yardimoglu, Department of Histology and Embryology, Faculty of Medicine, Kocaeli University, Campus of Umuttepe, Baki Komsuoglu Avenue, Kocaeli 41380, Turkey

E-mail: melda.yardimoglu@gmail.com

Abbreviations: CD26, dipeptidyl peptidase IV; IVF, in vitro fertilization; ART, assisted reproductive techniques; ET, embryo transfer; RIF, recurrent implantation failure; IW, implantation window; EVT, extravillous trophoblast; FSH, follicle-stimulating hormone; LT, luteinizing hormone; hCG, human chorionic gonadotrophin; $\mathrm{COH}$, controlled ovarian hyperstimulation; HBSS, Hanks' balanced salt solution; OPU, oocyte pick up; PBS, phosphate buffer saline; FITC, fluorescein isothiocyanate; DAPI, 4',6-diamidino-2-phenylindole; RGB, red/green/blue in Image $\mathrm{J}$ threshold; CAM, cell adhesion molecules

Key words: trophinin-dipeptidyl peptidase-embryo-endometrial co-culture-recurrent implantation failure the present study ( $n=5 / 13$ pregnancies). The average age of the successful group (28 \pm 3.54 years) was younger compared with the unsuccessful $(32.67 \pm 2.81)$ group $(\mathrm{P} \leq 0.05)$. The number of trophinin (+) endometrial cells in the presence of an embryo was significantly lower $(\mathrm{P}=0.046)$ in the successful group on the first day. No significant difference between the groups was observed in terms of the number of CD26 (+) cells on the first to the fourth days $(\mathrm{P} \leq 0.05)$. Trophinin and CD26 immunostaining is important in the early period of pregnancy, and it will be beneficial in terms of providing the deficit of conventional culture medium in performed studies with the endometrial co-culture medium. The co-culture may be important, particularly in the early period, in patients with recurrent implantation failure in terms of enabling a connection between the cells belonging to the endometrium and the embryo.

\section{Introduction}

In vitro fertilization (IVF) failure, and recurrent IVF failure, are known problems for couples (1-3). Endometrial receptiveness has an important role in IVF embryo transfer (ET) treatments, and a lack of consistency between embryo development and endometrial ripening causes failure $(3,4)$. The luminal epithelium is responsible for non-receptivity in the expression, organization or activation of adhesion systems (4). Implantation is affected by numerous variables involved in recurrent implantation failure (RIF). It has been suggested that this process may be hampered if either of these variables is defective. RIF is diagnosed when high-quality embryos repeatedly fail to implant following transference in several IVF treatment cycles (5). Chromosomal abnormalities, sperm DNA damage and inadequate culture conditions are all of importance in the etiology of RIF (6). It was observed that ovarian hyperstimulation itself is a factor for reduced endometrial receptivity (7). The Vero co-culture system is considered to be useful for IVF in terms of prolonging in vitro culture and enabling the transfer of embryos, as well as eliminating early-blocked eggs and freezing embryos at 
the blastocyst stage (8). Successful implantation requires the appropriately timed arrival of a viable blastocyst into a receptive endometrium. The endometrium is remodeled throughout the menstrual cycle, and exhibits a short period of receptivity, known as the 'implantation window' (IW) (9). Poor embryo quality has been identified as a major cause of implantation failure (10).

Cell adhesion molecules (CAMs) have been determined to serve specific roles in various phases in reproductive physiology. The functions of CAMs have been reported in biological and pathological states, and require cursory examination (11).

Trophinin is an intrinsic membrane protein, and its marked expression has been detected in the trophectoderm surface of monkey blastocysts. Expression of trophinin has also been observed in human endometrial surface epithelium on day $16 / 17$ at the early secretory phase, the time consistent with that expected for the IW (12). Trophinin is synthesized in implantation-associated cells of humans and primates, and is expressed in a restricted area of the human endometrial luminal epithelium during the early secretory phase. Restricted, but marked expression of trophinin in the IW indicates the specific role of trophinin throughout implantation in humans (12-14). In spite of this, trophinin is not limited to cells that are associated with implantation. Trophinin has been detected in the luminal and glandular epithelium of the endometrium, whether or not it includes the implanted blastocyst (15). Trophinin mediates homophilic and apical cell adhesion between trophoblastic cells and endometrial epithelial cells, which is potentially the initial attachment step in human embryo implantation (16). Trophinin is a dual signaling molecule. In embryonic cells, it promotes proliferation and invasion, whereas in maternal cells it promotes cell death in order to accept the invading embryo (17).

Dipeptidyl peptidase IV (CD26) is a membrane-binding extracellular glycoprotein expressed on extravillous trophoblasts (EVTs) at the decidua, and its enzymic activation leads to EVT invasion in women throughout the IW. It is known as an indicator molecule for the endometrium implantation phase expressed on the cell surface, and it can be reduced to various biological active peptidases in extracellular domains $(18,19)$. Overexpression of CD26 has been stated to cause a high blastocyst adhesion rate and high outgrowth domain in the trophectoderm (19). Therefore, the present study assessed the expression levels of trophinin and CD26 by immunofluorescence in embryos from the zygote to the blastocyst stage in co-culture medium of endometrial cells obtained from women patients with RIF.

\section{Materials and methods}

Patients. Statements of approval were received from the participants of the present study and the study was approved by the Kocaeli University Human Research Ethical Committee (approval no. KOU HREC:4/20; 2009 Feb 10/30). A total of 13 patients with RIF, aged between 26 and 36 years, were included in the present study. Common factors, including uterine polyps, leiomyomata and endometriosis, besides hormonal pathology, were excluded prior to the study by the gynecologist. Basal (menstrual day 3) levels of follicle-stimulating hormone (FSH), luteinizing hormone ( $\mathrm{LH})$, estradiol, thyroid-stimulating hormone, free triiodothyronine, free thyroxine and prolactin precursor were measured prior to the patients undergoing pituitary desensitization, followed by gonadotrophin ovarian stimulation and IVF treatment. Endometrial biopsies were obtained from the patients on day 21 of their menstrual cycle, termed the luteal phase. Controlled ovarian hyperstimulation $(\mathrm{COH})$ was performed in the patients to obtain plenty of oocytes. Long protocol was selected for decreasing cycle and sufficient ovarium response (poor response to ovulation induction results in increasing of cycle cancellation and implantation failure). In the same cycle, the patients were administered Lucrin depot (leuprolid asetat, 3M, $11.25 \mathrm{mg}$; AbbVie, Chicago, IL, USA) on day 21 and human chorionic gonadotrophin (hCG; Sigma Chemical Co., St. Louis MO, USA) on day 2 of menstrual bleeding. The dose was decreased to $5 \mathrm{mg} \mathrm{hCG}$ per injection when at least three follicles were $>17 \mathrm{~mm}$ in diameter.

Endometrial co-culture. Autologous endometrial co-culture was performed, as described previously (20). Briefly, a patient's fertilized eggs were placed on top of a layer of cells from her own uterine lining, creating a more natural environment for embryo development. Endometrial biopsies containing Hanks' balanced salt solution (HBSS) with 5\% penicillin-streptomycin-amphotericin (dilution: 5,000 $\mu \mathrm{g} / 100 \mathrm{ml}$; Biological Industries, Ltd., Kibbutz Beit Haemek, Israel) were obtained. In $15 \mathrm{~mm}$ centrifuge tubes, endometrial cells were isolated by digesting with $0.5 \%$ collagenase type II (Sigma-Aldrich, St. Louis, MO, USA) in $\mathrm{Ca}^{2+} / \mathrm{Mg}^{2+}$-free HBSS at $37^{\circ} \mathrm{C}$ for $5 \mathrm{~min}$. These cells were seeded onto poly-L-lysine coated four-well chamber slides (Thermo Fisher Scientific, Inc., Waltham, MA, USA). Endometrial cells were allowed to proliferate in the presence of recombinant human basic fibroblast growth factor (10 ng/ml; Biological Industries, Ltd.) in serum-free medium. The implantation was performed by putting lamella into two pieces of the four-well slide in order that each pit contained 500,000 cells. The patients' names and their dates of birth were written on the Petri dish as identifiers. The following day, after washing the cells with fresh medium, the solution was replaced and its top was covered with oil. Prepared as 5 pieces, one of the 4-well petries were suspended as in non-lubricated manner in the incubator for control endometrial co-culure. The cell culture procedure was performed in a laminar flow hood under sterile conditions.

Conventional culture protocol. All manipulations of oocytes and embryos were performed as previously described (1). The zygotes were cultured in a protein-free medium and cultured for $96 \mathrm{~h}$ (Sage IVF Inc., Trumbull, CT, USA) in a laminar flow hood under sterile conditions. The identical process was applied, and blastocysts were visualized on an inverted microscope (DMI 4000; Leica Microsystems GmbH, Wetzlar, Germany).

Oocyte pick up (OPU) and intracytoplasmic sperm injection. Two days after the hCG injection, the OPU process was performed in a sterile tube, accompanied by an OPU injection fixed in the ultrasonography probe, and collected oocytes were taken to the embryology laboratory. Oocytes in the sterile tube were taken by means of disposable sterile glass pipettes 
from folliculin liquid in a sterile container under the stereomicroscope (SZX7; Olympus Corporation, Tokyo, Japan) in the embryology laboratory. The cumulus cells around the eggs, obtained as a result of the OPU procedure, were cleared up, and subsequently the mature cells available for use were determined. For the microinjection procedure, the oocyte was located, and the sperm exhibiting a normal appearance in terms of form, and if available, liveliness, was selected under an inverted microscope. The sperm was inactivated by pressing in the middle of its tail by means of a microinjection pipette. The sperm was injected into the oocyte, fixed with a holding pipette. This procedure was performed for all oocytes in turn, with subsequent incubation at $37^{\circ} \mathrm{C}$ of the eggs in an incubator (MCO-18 M; Sanyo, Tokyo, Japan) and performance of a fertilization check $18-20 \mathrm{~h}$ after the procedure.

Culture environments and days. After taking a reading under an inverted microscope, the majority of the embryos were moved into a conventional culture environment $(n=80)$, the others into an endometrial co-culture environment $(n=25)$ on day 1 following OPU. Images of the embryo were captured using an inverted microscope (Olympus, Tokyo, Japan).

Day 1 after OPU. The RPMI-1640 medium (Sigma-Aldrich) in four-well dishes was refreshed three to five times, and loose dead cells, unlysed cells and erythrocytes were removed from the culture. The medium was prepared with human serum albumin (HSA; 1:10 in 0.75 ml; Life Global Medium) and was added into the wells, covered with $0.5 \mathrm{ml}$ oil and incubated. The endometrial cells were fixed with cold methanol on four-well dishes following ET to another co-culture.

Days 2-5 after OPU. The development of embryos was checked and recorded daily. The endometrial co-cultures were washed with new medium in the morning. The same process was applied between days 2-5.

Day 5 after $O P U$. The high-quality embryos were transferred, and endometrial cells were fixed with cold methanol on four-well dishes after ET.

The control endometrial co-cultures were fixed on four-well dishes without embryos and immunofluorescence staining was performed, as described below.

ET. Good-quality embryos were transferred with distended urinary bladder, using ultrasonography to determine the most appropriate place where the embryos would implant into the uterus. During ET, a speculum was placed in the vagina, and the cervix was cleansed with a sterile saline solution, and cervical mucus was cleaned with a sterile stick. The embryos were transferred into the uterus with the aid of a thin and soft catheter. Since the edges of the catheter can be monitored with ultrasonography, the area in the uterus where the embryos were transferred to was clearly determined. Loaded to the catheter in a laminar flow hood by an embryologist, the ET was performed gently by a surgeon, and the catheter was removed slowly and controlled under the microscope by the embryologist to determine whether all embryos were transferred or not. If present, any developing embryos not used in the transfer were frozen, according to their quality, or destroyed.
Immunocytochemistry. Endometrial cells were stained using an indirect immunofluorescent technique, similarly to a previously described protocol (19). Briefly, during the fixation process, the coverslip on the endometrial co-culture Petri dishes was removed. Endometrial cells were rinsed briefly in phosphate-buffered saline (PBS) and fixed in cold methanol for $10 \mathrm{~min}$. The coverslips were then allowed to dry completely. Following permeabilization with $0.025 \%$ Triton X-100 (Merck, Darmstadt, Germany), endometrial cells were incubated with $1.5 \%$ normal goat blocking serum (Santa Cruz Biotechnology, Inc., Santa Cruz, CA, USA) in PBS for $30 \mathrm{~min}$ at $37^{\circ} \mathrm{C}$ to suppress non-specific binding of immunoglobulin (Ig)Gs. Following washing three times with PBS (5 min each), the endometrial cells were incubated overnight at $4{ }^{\circ} \mathrm{C}$ with primary antibodies against rabbit anti-human CD26 (H-270; cat. no. SC-9153; Santa Cruz Biotechnology, Inc.) and mouse anti-human trophinin (cat. no. SC-80002; Santa Cruz Biotechnology, Inc.) at dilutions of 1:100 in PBS supplemented with 1\% (w/v) bovine serum albumin (Santa Cruz Biotechnology, Inc.). After three PBS washes, the cells were incubated with a 1:100 dilution of either goat anti-mouse IgM conjugated to fluorescein isothiocyanate (FITC; cat. no. SC-2082; Santa Cruz Biotechnology, Inc.) or a 1:100 dilution of goat anti-rabbit IgG conjugated to Texas red (cat. no. SC-2780; Santa Cruz Biotechnology, Inc.) secondary antibodies for $25 \mathrm{~min}$ in the dark. Following washing three times with PBS, the cells were mounted with mounting medium containing 4',6-diamidino-2-phenylindole (DAPI; $1 \mathrm{mg} / \mathrm{ml}$; Santa Cruz Biotechnology, Inc.) to counterstain the nucleus. A negative control of immunofluorescence staining was incubated with PBS as a primary antibody and then secondary antibody to determine any non-specific binding. Immunofluorescent staining was observed as green with FITC for trophinin, and red with Texas red for CD26, using an inverted wide-field fluorescence microscope. Images were captured using a Leica camera (DMI 4000B; Microsystems $\mathrm{GmbH}$ ).

Enumeration of cells in the micrograph. In each endometrial co-culture Petri dish $(n=4)$ of each patient, quantification of positive immunofluorescence staining was performed in $0.20 \mathrm{~mm}^{2}$ fields with a $\mathrm{x} 40$ objective, using Image $\mathrm{J}$ version 1.44a software with Java ${ }^{\mathrm{TM}}$ by National Institutes of Health (Bethesda, MD, USA). Endometrial cells were examined by two independent observers in a blinded manner, using the Image J software for analysis and digitization (21). Looping cell enumeration was used on images obtained for groups by the Fero lab (Fred Hutchinson Cancer Research Center, Seattle, WA, USA), and Image $\mathbf{J}$ for analysis and digitization.

Statistical analysis. The data were analyzed using SPSS software version 13.0 (SPSS, Inc., Chicago, IL, USA) for Windows. The Spearman test for correlation analysis among ongoing variables, the Mann Whitney-U test to identify the difference between each group, the Wilcoxon signed-rank test for the comparison of two associated samples, and the Friedman test to compare all groups were performed. $\mathrm{P}<0.05$ was considered to indicate a statistically significant difference. The data are presented as the mean \pm standard deviation. 


\section{Results}

Patients and embryos. The age, basal hormonal values and endometrial co-cultures were evaluated in 11 patients. No difference in terms of the number of pregnancies or embryo development were observed between each culture environment. The embryos of 11 women had developed normally, with the exception of 2 women from the 13 women involved in the present study, the embryos were arrested in 2 patients. No difference was observed on the first to the fourth days between the two culture groups in terms of the grade (cell number, irregular blastomeres, fragmentation, multinucleation of embryo). In the unsuccessful group with implantation failure on the third day, the morphology of morula in the endometrial co-culture (Fig. 1A) and conventional co-culture (Fig. 1B) was identical. The number of cells was important in embryo development for pregnancy. These eight-cell embryos were moderately fragmented and had irregular cells on the third day. In the successful pregnancy group, on same day, the microscopic appearance of the morula in endometrial co-culture (Fig. 1C) and conventional co-culture (Fig. 1D) exhibited no morphological difference.

The patients were grouped according to development of the embryo, implantation and pregnancy status as either unsuccessful, unsuccessful with implantation failure or successful pregnancy groups.

Unsuccessful groups. Patients in the unsuccessful group exhibited developmentally arrested embryos and non-implanted embryos. These embryos failed to develop, and pregnancy was unsuccessful in these patients $(n=2)$.

Unsuccessful group with implantation failure. Patients in this group exhibited well-developed embryos; however, implantation failure was observed $(n=6)$. Although the developed embryos were transferred, due to implantation failure, pregnancy was unsuccessful in this group.

Successful pregnancy group. Patients exhibited well-developed embryos and appeared pregnant, with successful implantation $(n=5)$. The developed embryos were transferred and pregnancy occurred in this group.

While the average age of women involved in the present study was $28 \pm 3.54$ in the successful group, the average age in the unsuccessful group was $32.67 \pm 2.81$. A difference between the average ages of the groups was clear; however, no difference was observed between the basal hormonal values in each group $(\mathrm{P}=0.035$; Table $\mathrm{I})$. The ratio of successful pregnancy was $0.38(n=5 / 13)$.

Immunofluorescence staining was performed for the co-culture: Trophinin (+) cells were yellow-green (FITC), CD26 (+) cells were red (Texas red) and the nuclei were blue with DAPI, on endometrial co-culture cells (Fig. 2). No data regarding trophinin and CD26 (+) cells were obtained for the co-culture of the unsuccessful group as a result of arrested embryos. The number of trophinin and CD26 (+) cells were observed on the first to the fourth days of embryo development in both culture groups. A significant difference was observed in the number of trophinin $(+)$ cells on the first day between each group $(\mathrm{P}=0.046)$. The number of CD26 $(+)$ cells
Table I. Age and basal hormonal values in the unsuccessful and the successful pregnancy groups.

\begin{tabular}{lccc}
\hline \multicolumn{3}{c}{ Group } \\
\cline { 2 - 3 } Parameter & $\begin{array}{c}\text { Unsuccessful } \\
(\mathrm{n}=6)\end{array}$ & $\begin{array}{c}\text { Successful } \\
(\mathrm{n}=5)\end{array}$ & \\
\hline Age & $32.67 \pm 2.81$ & $28.00 \pm 3.54$ & $0.035^{\mathrm{a}}$ \\
FSH & $6.33 \pm 1.15$ & $7.26 \pm 2.54$ & 0.583 \\
LH & $4.52 \pm 1.16$ & $4.71 \pm 0.34$ & 0.783 \\
E2 & $46.78 \pm 16.44$ & $36.62 \pm 5.60$ & 0.465 \\
TSH & $2.53 \pm 1.33$ & $1.42 \pm 0.75$ & 0.054 \\
FT3 & $3.38 \pm 0.21$ & $3.61 \pm 0.25$ & 0.198 \\
FT4 & $1.33 \pm 0.29$ & $1.37 \pm 0.15$ & 0.279 \\
PRL & $23.18 \pm 5.19$ & $18.88 \pm 13.55$ & 0.273 \\
\hline
\end{tabular}

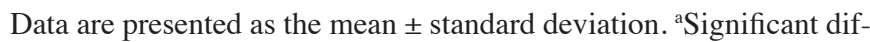
ference vs. unsuccessful group. FSH, follicle-stimulating hormone; LH, luteinizing hormone; E2, estradiol; TSH, thyroid-stimulating hormone; FT3, free triiodothyronine; FT4, free thyroxine; PRL, prolactin precursor.

was higher, with the exception of the third to the fourth days, and trophinin (+) cells were lower in the successful group. No difference was observed between the number of trophinin and CD26 (+) cells on the second to the fourth days. Additionally, the number of control CD26 (+) cells were higher in the successful group (Table II; Figs. 2 and 3). A negative correlation was determined between control CD26 and trophinin parameters $(\mathrm{r}=-0.836 ; \mathrm{P}=0.005$; Table II). A positive correlation between ages and the number of CD26 (+) cells was observed on the third day $(\mathrm{r}=0.678 ; \mathrm{P}=0.045)$. A positive correlation was also observed between the first and second days in the number of CD26 $(+)$ cells $(r=0.817 ; \mathrm{P}=0.007)$ and between the first day number of CD26 (+) cells and the fourth day number of trophinin $(+)$ cells $(r=0.763 ; \mathrm{P}=0.017$; $\mathrm{P}<0.05)$. Therefore, the highest $(+)$ cell numbers in the number of CD26 and trophinin (+) cells were on the fourth day. An inequality between the number of control trophinin $(+)$ cells on the first $(\mathrm{P}=0.036)$ and second days $(\mathrm{P}=0.021 ; \mathrm{P}<0.05)$ was associated with the number of trophinin $(+)$ cells. Briefly, the number of trophinin $(+)$ cells on the first and second days was lower compared with the control trophinin $(+)$ cells (Table II; Figs. 2 and 3).

\section{Discussion}

Observing no difference between the basal hormone levels in each group in the present study may be due to the patients with RIF. Serum FSH and LH were known prognostic indicators on the second day in the treatment with IVF, and FSH was particularly useful in predicting the ovarian response to superovulation (22). The basal FSH concentration is also known as a better predictor of the cancellation rate and of the number of oocytes collected in IVF treatment compared with age; however, age was a stronger predictor of the pregnancy rate (23). 
Table II. Quantification of the number of trophinin and CD26 (+) cells in the absence or presence of embryos on the first to the fourth days in the endometrial co-culture of the unsuccessful and the successful pregnancy groups.

\begin{tabular}{|c|c|c|c|}
\hline \multirow[b]{2}{*}{ Immunoreactivity of ECs } & \multicolumn{2}{|c|}{ Group } & \multirow[b]{2}{*}{ P-value } \\
\hline & $\begin{array}{l}\text { Unsuccessful } \\
\qquad(\mathrm{n}=6)\end{array}$ & $\begin{array}{l}\text { Successful } \\
\quad(n=5)\end{array}$ & \\
\hline \multicolumn{4}{|l|}{ Control } \\
\hline Trophinin ECs ${ }^{\mathrm{b}}$ & $25.40 \pm 15.82$ & $17.25 \pm 19.76$ & 0.140 \\
\hline $\mathrm{CD} 26 \mathrm{ECs}^{\mathrm{b}}$ & $22.00 \pm 9.77$ & $30.75 \pm 12.92$ & 0.268 \\
\hline \multicolumn{4}{|l|}{ Day 1} \\
\hline Trophinin $\mathrm{ECs}^{\mathrm{c}}$ & $11.00 \pm 4.30^{\mathrm{a}}$ & $5.00 \pm 1.63^{\mathrm{a}}$ & $0.046^{\mathrm{a}}$ \\
\hline CD26 ECs ${ }^{c}$ & $17.60 \pm 5.32$ & $32.00 \pm 25.86$ & 0.624 \\
\hline \multicolumn{4}{|l|}{ Day 2} \\
\hline Trophinin ECs $s^{c}$ & $9.20 \pm 7.43$ & $5.00 \pm 2.16$ & 0.537 \\
\hline $\mathrm{CD} 26 \mathrm{ECs}^{\mathrm{c}}$ & $20.80 \pm 9.15$ & $25.25 \pm 12.34$ & 0.462 \\
\hline \multicolumn{4}{|l|}{ Day 3} \\
\hline Trophinin $\mathrm{ECs}^{\mathrm{c}}$ & $16.40 \pm 11.78$ & $3.50 \pm 2.38$ & 0.138 \\
\hline $\mathrm{CD} 26 \mathrm{ECs}^{\mathrm{c}}$ & $28.00 \pm 11.77$ & $18.50 \pm 17.62$ & 0.221 \\
\hline \multicolumn{4}{|l|}{ Day 4} \\
\hline Trophinin ECs $s^{c}$ & $10.00 \pm 9.46$ & $9.25 \pm 11.33$ & 0.803 \\
\hline $\mathrm{CD} 26 \mathrm{ECs}^{\mathrm{c}}$ & $38.00 \pm 34.91$ & $27.00 \pm 19.20$ & 0.806 \\
\hline
\end{tabular}

Data are presented as the mean \pm standard deviation. a Significant difference vs. unsuccessful group. Immunoreactivity of endometrial co-culture cells in the ${ }^{\mathrm{b}}$ absence or ${ }^{\mathrm{c}}$ presence of embryo. CD26, dipeptidyl peptidase IV; EC, endometrial cell.
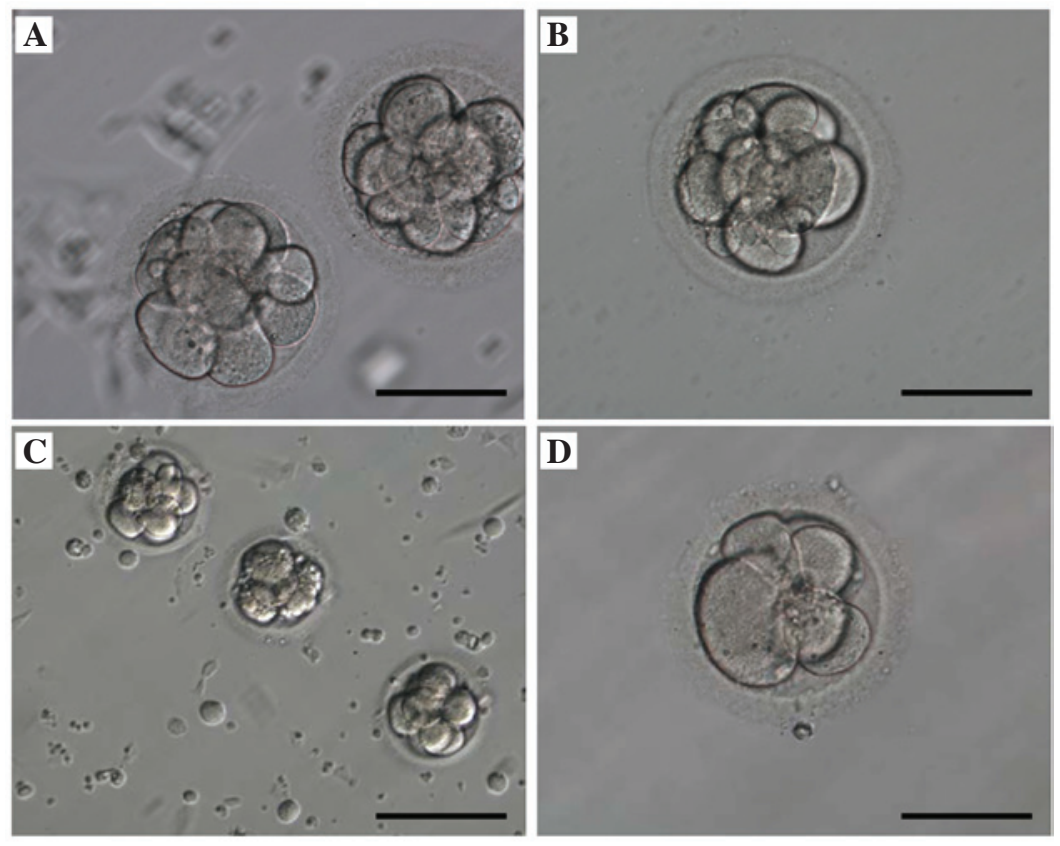

Figure 1. Embryos are shown in culture media. Embryos on the third day for the (A and B) unsuccessful pregnancy group and (C and D) successful pregnancy group are shown, in the (A and C) endometrial co-culture and in (B and D) conventional culture (scale bar, $50 \mu \mathrm{m}$ ).

Autologous endometrial co-culture, more commonly known as co-culture, is a state-of-the-art technique co-developed by Abington Reproductive Medicine's Dr. Larry Barmat (20). This procedure has a more natural environment for embryo development and maximizes the chance for a successful IVF pregnancy. It is known that co-culture may be an effective treatment for patients who have failed previous IVF cycles, or who have poor embryo quality (20). The quality of embryos in autologous endometrial co-culture has been determined to be better than that of embryos in non-co-culture (24). No 

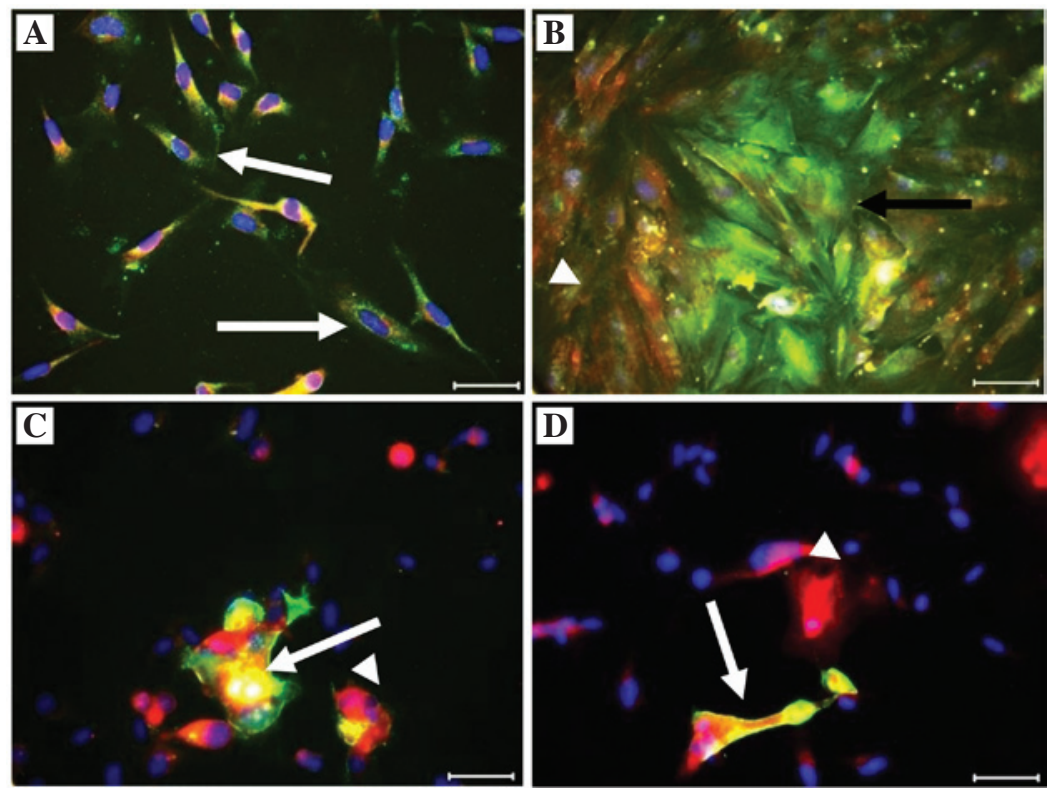

Figure 2. Double immunofluorescence staining photomicrographs of the unsuccessful pregnancy group. (A) Control, (B) first day, (C) third day and (D) fourth day of the endometrial co-culture in the unsuccessful pregnancy group. The double staining of trophinin (yellow/green) and CD26 (red) revealed co-localization with different quantities in the presence of embryos on the first and the fourth days. Nuclei were counterstained with 4',6-diamidino-2-phenylindole (blue). The arrows indicate trophinin (+) cells and the arrow heads indicate CD26 (+) cells (scale bar, $50 \mu \mathrm{m})$.
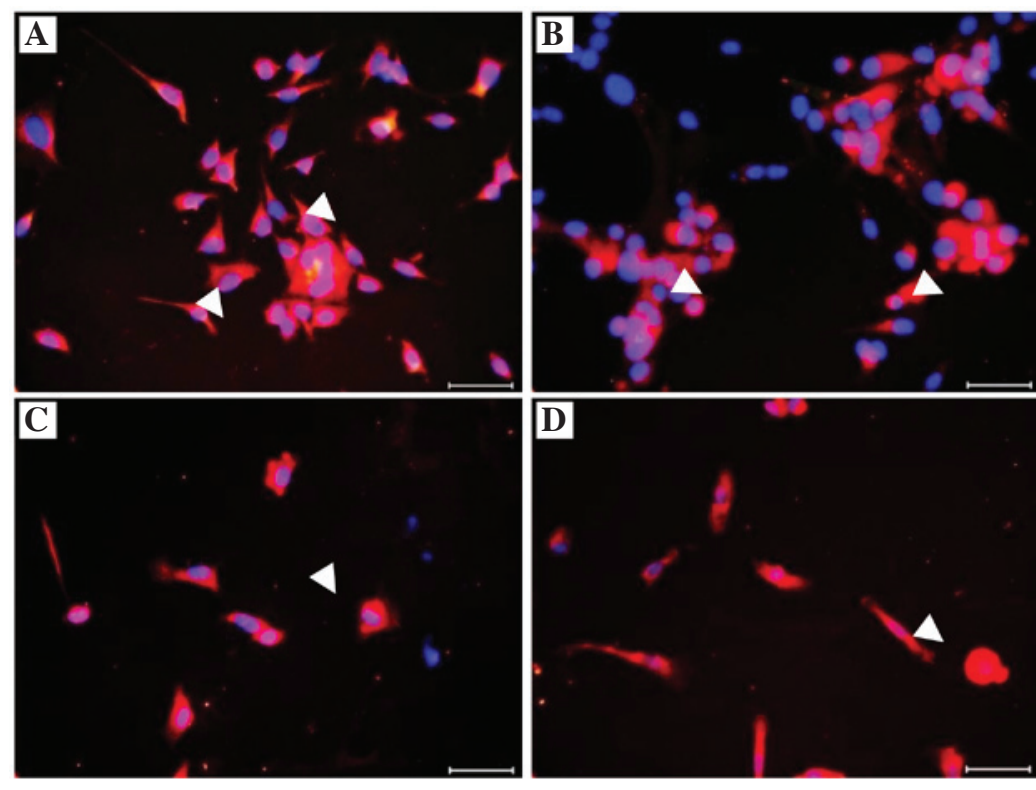

Figure 3. Double immunofluorescence staining photomicrographs of the successful pregnancy group. (A) Control, (B) first day, (C) third day and (D) fourth day of the endometrial co-culture in the successful pregnancy group. Double staining of trophinin and CD26 revealed that red CD26 positivity is dominant in the presence of embryo on the first and the fourth days. Nuclei were counterstained with 4',6-diamidino-2-phenylindole (blue). Arrow heads indicate CD26 (+) cells (scale bar, $50 \mu \mathrm{m}$ ).

difference in terms of embryo quality was observed between the two culture environments, due to the development of mediums. The difference between each group was significant in terms of the number of trophinin (+) and CD26 (+) cells, as control in the absence of an embryo on the first to the fourth days. The adhesion mechanism has been previously shown to be involved in human blastocyst implantation by endometrial CD26 (+) and embryonal fibronectin (19). IVF embryos developed in vitro in culture media, allowing them to be maintained alive for a longer period of time, has led to a rise in pregnancy rates (8). In the present study, at the blastocyst stage, the ET was possible due to continuation of pregnancy. A statistically significant difference was observed between the average ages of the groups. Age has been reported as a clear predictor of the pregnancy rate (23).

The successful group exhibited a higher number of CD26 (+) cells, with an exception on the third day. A significant difference was observed with regard to the first to the fourth days, the early growth phase, and the expression of trophinin (+) and CD26 (+) (P=0.046). The successful group 
exhibited a lower number of trophinin (+) and CD26 (+) cells in the controls, unlike the unsuccessful group. Since the trophoectoderm of the human blastocyst secretes hCG prior to and following implantation, these results suggested that hCG from the human embryo induces trophinin expression by maternal cells (25). Trophinin-mediated signal transduction has been described in trophectoderm cells and endometrial epithelial cells (17).

The highest cell numbers of CD26 (+) and trophinin (+) were on the fourth day. The expression of trophinin has been investigated in human blastomeres and blastocysts by immunofluorescence and laser scanning confocal microscopy. This expression was intensified in the course of embryonic development (26). Trophinin expression of endometrial cells was stronger in the control groups. However, no consistent increase or decrease of trophinin/CD26 (+) cells was obtained in the course of the pre-implantation embryos, since endometrial biopsies and oocytes were obtained from the patients with RIF.

In a clinical program with in vitro models, the embryos can be co-cultured with endometrial cells until the blastocyst stage, and subsequently transferred back into the mother (27). These models have provided information about embryonic regulation of endometrial epithelial molecules, including anti-adhesion molecules (28), cytoskeletal proteins (29) and chemokines (30), during human implantation. Structural and hormonal changes occur in blastocyst invasion, and these changes have been demonstrated using time-lapse photography, immunostaining and hCG levels for human-hatched blastocyst co-culture with human endometrial stromal cell monolayers (31).

Immunostaining of trophinin and CD26 suggested that endometrial co-culture cells may influence implantation with CAMs. It may be suitable, both in terms of enabling improvements of conventional culture medium with immunohistochemical studies performed in endometrial co-culture, and in providing connections in the early period among cells of the gravid endometrium and embryo in unexplained infertility. Natural growth factors, proteins and nutrients may support embryo development in the co-culture environment. Therefore, co-culture may be a considerable alternative for patients with RIF. It is important that the development of endometrial co-culture techniques is performed, instead of the conventional culture methods for patients with RIF.

\section{Acknowledgements}

The authors would like to thank Associate Professor Dr. Dogan Yuksel and lecturer Mrs Ayca Ozguven from Kocaeli University Department of Foreign Languages for editing of our manuscript. The present study was financially supported by the Scientific Research Foundation of Kocaeli University Scientific Research Foundation (no. 2007/70).

\section{References}

1. Gardner DK and Lane M: Culture systems for the human embryo. In: Gardner DK, Weissman A, Howles CM, Shoham Z, editors. Textbook of Assisted Reproductive Technologies Laboratory and Clinical Perspectives. 3rd ed. UK, Informa Healthcare, Taylor \& Francis; USA, 219-240, 2009.

2. Penzias AS: Recurrent IVF failure: Other factors. Fertil Steril 97: 1033-1038, 2012.
3. Revel A: Defective endometrial receptivity. Fertil Steril 97: 1028-1032, 2012.

4. Aplin JD: Embryo implantation: The molecular mechanism remains elusive. Reprod Biomed Online 14 Spec No 1: 49-55, 2007.

5. Laufer N and Simon A: Recurrent implantation failure: Current update and clinical approach to an ongoing challenge. Fertil Steril 97: 1019-1020, 2012.

6. Das M and Holzer HE: Recurrent implantation failure: Gamete and embryo factors. Fertil Steril 97: 1021-1027, 2012.

7. Liu L, Tong X, Jiang L, Li T, Zhou F and Zhang S: A comparison of implantation, miscarriage and pregnancy rates of single and double day 3 embryo transfer between fresh and frozen thawed transfer cycles: A retrospective study. Chin Med J (Engl) 127: 911-915, 2014.

8. Menezo YJ, Guerin JF and Czyba JC: Improvement of human early embryo development in vitro by coculture on monolayers of Vero cells. Biol Reprod 42: 301-306, 1990.

9. Moore KL, Persaud TVN and Torchia MG: First and second week of human development, Chapter: 2,3 . In: The Developing Human. Clinically Oriented Embryology. 9th ed. (International ed.), Elsevier Saunders, ISBN: 978-0-8089-2444-9; 32-51, 2013.

10. Urman B, Yakin K and Balaban B: Recurrent implantation failure in assisted reproduction: How to counsel and manage. A. General considerations and treatment options that may benefit the couple. Reprod Biomed Online 11: 371-381, 2005.

11. Atabekoğlu CS, Engin Y, Üstün Y and Aytaç R: Reproductive physiology and adhesion molecules. J Ankara Univ Faculty Med 55: 85-92, 2002 (In Turkish).

12. Fukuda MN, Sato T, Nakayama J, Klier G, Mikami M, Aoki D and Nozawa S: Trophinin and tastin, a novel cell adhesion molecule complex with potential involvement in embryo implantation. Genes Dev 9: 1199-1210, 1995.

13. Suzuki N, Zara J, Sato T, Ong E, Bakhiet N, Oshima RG, Watson KL and Fukuda MN: A cytoplasmic protein, bystin, interacts with trophinin, tastin, and cytokeratin and may be involved in trophinin-mediated cell adhesion between trophoblast and endometrial epithelial cells. Proc Natl Acad Sci USA 95: 5027-5032, 1998.

14. Suzuki N, Nadano D, Paria BC, Kupriyanov S, Sugihara K and Fukuda MN: Trophinin expression in the mouse uterus coincides with implantation and is hormonally regulated but not induced by implanting blastocysts. Endocrinology 141: 4247-4254, 2000.

15. Nadano D, Sugihara K, Paria BC, Saburi S, Copeland NG, Gilbert DJ, Jenkis NA, Nakayama J and Fukuda MN: Significant differences between mouse and human trophinins are revealed by their expression patterns and targeted disruption of mouse trophinin. Gene Biol Reprod 66: 313-321, 2002.

16. Aoyama J, Nakayama Y, Sugiyama D, Saburi S, Nadano D, Fukuda MN and Yamaguchi N: Apical cell adhesion molecule, trophinin, localizes to the nuclear envelope. FEBS Lett 579: 6326-6332, 2005.

17. Fukuda MN and Sugihara K: Cell adhesion molecules in human embryo implantation. Sheng Li Xue Bao 64: 247-258, 2012.

18. Sato Y, Fujiwara H, Higuchi T, Yoshioka S, Tatsumi K, Maeda M and Fujii S: Involvement of dipeptidyl peptidase IV in extravillous trophoblast invasion and differentiation. J Clin Endocrinol Metab 87: 4287-4296, 2002.

19. Shimomura Y, Ando H, Furugori K, Kajiyama H, Suzuki M, Iwase A, Mizutani S and Kikkawa F: Possible involvement of crosstalk cell-adhesion mechanism by endometrial CD26/dipeptidyl peptidase IV and embryonal fibronectin in human blastocyst implantation. Mol Hum Reprod 12: 491-495, 2006.

20. Barmat LI, Liu H, Spandorfer SD, Kowalik A, Mele C, Xu K, Veeck L, Damario M and Rozenwaks Z: Autologous endometrial co-culture in patients with repeated failures of implantation after in vitro fertilization-embryo transfer. J Assist Reprod Genet 16: 121-127, 1999.

21. Collins TJ: ImageJ for microscopy. Biotechniques 43 (1 Suppl): 25-30, 2007.

22. Chan YF, Ho PC, So WW and Yeung WS: Basal serum pituitary hormone levels and outcome of in vitro fertilization utilizing a flare nasal gonadotropin releasing hormone agonist and fixed low-dose follicle-stimulating hormone/human menopausal gonadotropin regimen. J Assist Reprod Genet 10: 251-254, 1993.

23. Sharif K, Elgendy M, Lashen $\mathrm{H}$ and Afnan M: Age and basal follicle stimulating hormone as predictors of in vitro fertilisation outcome. Br J Obstet Gynaecol 105: 107-112, 1998. 
24. Spandorfer SD, Soslow R, Clark R, Fasouliotis S, Davis OK and Rosenwaks Z: Histologic characteristics of the endometrium predicts success when utilizing autologous endometrial coculture in patients with IVF failure. J Assist Reprod Genet 23: 185-189, 2006.

25. Nakayama J, Aoki D, Suga T, Akama TO, Ishizone S, Yamaguchi H, Imakawa K, Nadano D, Fazleabas AT, Katsuyama $\mathrm{T}$, et al: Implantation-dependent expression of trophinin by maternal fallopian tube epithelia during tubal pregnancies: Possible role of human chorionic gonadotrophin on ectopic pregnancy. Am J Pathol 163: 2211-2219, 2003

26. Wang HY, Xing FQ and Chen SL: Expression of trophinin in human oocytes and preimplantation embryos. Nan Fang Yi Ke Da Xue Xue Bao 28: 122-124, 2008 (In Chinese).

27. Mercader A, Garcia-Velasco JA, Escudero E, Remohí J, Pellicer A and Simón C: Clinical experience and perinatal outcome of blastocyst transfer after coculture of human embryos with human endometrial epithelial cells: A 5-year follow-up study. Fertil Steril 80: 1162-1168, 2003.
28. Meseguer M, Aplin JD, Caballero-Campo P, O'Connor JE, Martín JC, Remohí J, Pellicer A and Simón C: Human endometrial mucin MUC1 is up-regulated by progesterone and down-regulated in vitro by the human blastocyst. Biol Reprod 64: 590-601, 2001.

29. Martin JC, Jasper MJ, Valbuena D, Meseguer M, Remohí J, Pellicer A and Simón C: Increased adhesiveness in cultured endometrial-derived cells is related to the absence of moesin expression. Biol Reprod 63: 1370-1376, 2000.

30. Dominguez F, Galan A, Martin JJ, Remohi J, Pellicer A and Simón C: Hormonal and embryonic regulation of chemokine receptors CXCR1, CXCR4, CCR5 and CCR2B in the human endometrium and the human blastocyst. Mol Hum Reprod 9: 189-198, 2003.

31. Carver J, Martin K, Spyropoulou I, Barlow D, Sargent I and Mardon H: An in-vitro model for stromal invasion during implantation of the human blastocyst. Hum Reprod 18: 283-290, 2003. 